\title{
A coupled interface-body nonlocal damage model for the analysis of FRP strengthening detachment from cohesive material
}

\author{
S. Marfia, E. Sacco, J. Toti \\ Department of Mechanics, Structures and Environment, University of Cassino, Cassino (Italy) \\ marfia@uncas.it,sacco@unicas.it,jessica.toti@unicas.it
}

\begin{abstract}
In the present work, a new model of the FRP-concrete or masonry interface, which accounts for the coupling occurring between the degradation of the cohesive material and the FRP detachment, is presented; in particular, a coupled interface-body nonlocal damage model is proposed. A nonlocal damage and plasticity model is developed for the quasi-brittle material. For the interface, a model which accounts for the mode I, mode II and mixed mode of damage and for the unilateral contact and friction effects is developed. Two different ways of performing the coupling between the body damage and the interface damage are proposed and compared. Some numerical applications are carried out in order to assess the performances of the proposed model in reproducing the mechanical behavior of the masonry elements strengthened with external FRP reinforcements.
\end{abstract}

SOMMARIO. Nel presente lavoro si propone un modello di interfaccia FRP-calcestruzzo o FRP-muratura, che tiene conto dell'accoppiamento tra il degrado del materiale coesivo ed il distacco del FRP; in particolare, si sviluppa un modello di danno non locale accoppiato interfaccia-corpo. Si presenta un modello di danno non locale e plasticità per il materiale coesivo ed un modello di interfaccia che tiene conto del modo I, II e misto di danno, del contatto unilatero e degli effetti dell'attrito. Si propongono e confrontano due diversi modi di accoppiamento del danno del corpo e del danno d'interfaccia. Si sviluppano applicazioni numeriche per verificare l'efficienza del modello proposto nel riprodurre il comportamento meccanico di elementi in muratura rinforzati con FRP.

KEYWORDS. Interface-body damage; Detachment phenomenon; Nonlocal model.

\section{INTRODUCTION}

7 he use of Fiber Reinforced Plastic (FRP) materials for the strengthening of existing concrete and masonry elements is growing; in the last twenty years, many structures have been reinforced adopting FRP and several experimental and modeling scientific works have been developed [1-9]. The use of FRP materials applied on the external surface of concrete or masonry structures aroused new modeling problems. One of the main problem in the use of FRP is the detachment phenomenon, which consists in the sudden decohesion of the FRP reinforcement from the concrete or masonry surface.

Indeed, the concrete and the masonry are quasi-brittle materials, whose mechanical response is characterized by damage with softening, which is due to the development of micro-cracks. Thus, two damage effects could be presented in the quasi-brittle reinforced structural elements: the body damage, which develops inside the domain of the strengthened element, and the interface damage, which occurs at the FRP -concrete or -masonry interface. Experimental evidences demonstrate that the detachment of the FRP from the support material occurs often with peeling of a thin layer from the 
external surface of the quasi-brittle material; this collapse behavior is due to the fact that the tensile and shear strength of the glue used to apply the FRP to the support is generally greater than the strength of the concrete or masonry support. From this observation, it can be deduced that the body damage and the interface damage cannot evolve independently one from the other; in other words, their evolution is coupled [10].

In the present work, a new model of the FRP-concrete or masonry interface, that takes into account the coupling occurring between the degradation of the cohesive support material and the FRP detachment, is presented. A nonlocal damage and plasticity model is developed for the cohesive support material. An interface model which accounts for the mode I, mode II and mixed mode of damage and for the unilateral contact and friction effects is developed. Two different ways of performing the coupling between the body and the interface damage are proposed. Both the approaches assume that the interface damage is influenced not only by the detachment stresses but also by the body damage computed on the bond surface. The first approach ensures that the interface damage is not lower than the body damage evaluated at the bond surface [11]. The second approach is based on simplified micromechanical considerations. Some numerical applications are performed in order to assess the performances of the proposed coupled interface models in reproducing the mechanical behavior of the masonry elements strengthened with external FRP reinforcements.

\section{A COUPLED BODY-INTERFACE DAMAGE MODEL}

he structural system, schematizing the FRP reinforced concrete or masonry element, is studied in the framework of two-dimensional plane stress elasticity, considering small strain and displacement regime. The system, consists in three subsystems: the body $\Omega_{1}$, modeling the concrete or masonry element, characterized by a cohesive constitutive law; the body $\Omega_{2}$, modeling the FRP reinforcement, characterized by a linear elastic behavior; the interface $\mathfrak{I}$ , modeling the connection between the reinforcement and the cohesive support material, characterized by a damaging behavior with friction and unilateral contact effects.

In particular, the interface $\mathfrak{I}$ is assumed to be constituted by three layers:

$\checkmark$ the glue, whose mechanical properties are generally much better than those of the support cohesive material;

$\checkmark$ a thin layer of the support cohesive material in which, during the application of the reinforcement, the glue penetrates the pores, improving its mechanical properties;

$\checkmark$ a further thin layer of the support cohesive material in which the detachment process occurs.

Indeed, the first two layers remain joined to the FRP after the complete detachment of the reinforcement. The interface damaging process, occurring in the third layer, can be due to the stress induced by the detachment action and also by the degradation of the support cohesive material. As a consequence, the damage occurring in the body $\Omega_{1}$ influences the behavior and the detachment process of the interface. On the contrary, it can be assumed that the damage of the third layer, generated by the detachment stresses, remains localized in the interface, i.e. it does not influences the body damage. In order to take into account these two possible damaging effects, an interface coupled damage model should be adopted. In fact, the coupling ensures that the damage evolution in the interface depends on the body damage and not vice-versa.

The constitutive laws of the body $\Omega_{1}$, of the interface $\mathfrak{I}$, neglecting the coupling between the body and the interface damage, and of the new proposed interface $\mathfrak{I}$, considering two different ways of coupling the body and interface degradation, are presented in the following.

\section{Body nonlocal damage model for the cohesive material}

A plastic nonlocal damage model, characterized by the following constitutive law, is considered for the body $\Omega_{1}$ :

$$
\boldsymbol{\sigma}^{\Omega}=\overline{\boldsymbol{\sigma}}^{\Omega}\left[\left(1-D_{t}^{\Omega}\right) H\left(\operatorname{sgn}\left(\operatorname{tr}\left(\mathbf{e}^{\Omega}\right)\right)\right)+\left(1-D_{c}^{\Omega}\right)\left(1-H\left(\operatorname{sgn}\left(\operatorname{tr}\left(\mathbf{e}^{\Omega}\right)\right)\right)\right]\right.
$$

with $\boldsymbol{\sigma}^{\Omega}$ the stress tensor, $D_{t}^{\Omega}$ and $D_{c}^{\Omega}$ the damage variables in tension and in compression, respectively, the symbol $\operatorname{sgn}(\bullet)$ indicating the sign of the variable $\bullet, H(\bullet)$ the Heaviside function, i.e. $H(\bullet)=1$ if $\bullet \geq 0$, otherwise $H(\bullet)=0$, and $\overline{\boldsymbol{\sigma}}^{\Omega}$ the effective stress defined as:

$$
\overline{\boldsymbol{\sigma}}^{\Omega}=\mathbf{C}^{\Omega}\left(\boldsymbol{\varepsilon}^{\Omega}-\boldsymbol{\varepsilon}_{p}^{\Omega}\right)=\mathbf{C}^{\Omega} \mathbf{e}^{\Omega}
$$


where $\mathbf{C}^{\Omega}$ is the elastic tensor, $\boldsymbol{\varepsilon}^{\Omega}, \boldsymbol{\varepsilon}_{p}^{\Omega}$ and $\mathbf{e}^{\Omega}$ are the total strain, the plastic strain and the elastic strain tensors, respectively.

The following plastic yield function is introduced:

$$
f\left(\overline{\boldsymbol{\sigma}}^{\mathbf{\Omega}}\right)=A-\left(\bar{\sigma}_{1}-\sigma_{y}\right)\left(\bar{\sigma}_{2}-\sigma_{y}\right)+B\left(\left(\left\langle\bar{\sigma}_{1}-\sigma_{y}\right\rangle_{-}\right)^{2}+\left(\left\langle\bar{\sigma}_{2}-\sigma_{y}\right\rangle_{-}\right)^{2}\right)
$$

with $\bar{\sigma}_{1}$ and $\bar{\sigma}_{2}$ the principal stresses of the effective stress tensor $\overline{\boldsymbol{\sigma}}^{\mathbf{\Omega}}, \sigma_{y}$ the yield stress and $A$ and $B$ material parameters governing the shape of the yield function (a brunch of hyperbola). In particular, it is set $A=0.1 \mathrm{MPa}$ and $B=100$. The evolution law of the plastic strain is:

$$
\dot{\boldsymbol{\varepsilon}}_{p}^{\mathbf{\Omega}}=\dot{\lambda} \frac{\partial f}{\partial \overline{\boldsymbol{\sigma}}^{\mathbf{\Omega}}}
$$

which is completed with the classical loading-unloading Kuhn-Tucker conditions:

$$
\dot{\lambda} \geq 0, \quad f\left(\overline{\boldsymbol{\sigma}}^{\Omega}\right) \leq 0, \quad \dot{\lambda} f\left(\overline{\boldsymbol{\sigma}}^{\Omega}\right)=0
$$

The accumulated plastic strain $\kappa^{\Omega}$ is introduced as $\kappa^{\Omega}=\int_{0}^{t}\left\|\dot{\boldsymbol{\varepsilon}}_{p}^{\Omega}\right\| d t$.

As a damage softening constitutive law is introduced, localization of the strain and damage parameter could occur. In order to overcome this pathological problem, to account for the correct size of the localization zone and, also, to avoid strong mesh sensitivity in finite element analyses, a nonlocal constitutive law is considered. In particular, an integral nonlocal model is adopted for the damage in compression and in tension.

The evolution of the compressive damage variable is governed by the following law:

$$
D_{c}^{\Omega}=\max _{\text {history }}\left\{\min \left\{1, \tilde{D}_{c}^{\Omega}\right\}\right\} \quad \tilde{D}_{c}^{\Omega}=-\frac{2}{\varepsilon_{u}^{3}}\left(\bar{\kappa}^{\Omega}\right)^{3}+\frac{3}{\varepsilon_{u}^{2}}\left(\bar{\kappa}^{\Omega}\right)^{2}
$$

with $\varepsilon_{u}$ the final damage threshold in compression and $\bar{\kappa}^{\Omega}$ the nonlocal accumulated plastic strain, evaluated at the point $\mathbf{x}$, as:

$$
\bar{\kappa}^{\Omega}(\mathbf{x})=\frac{1}{\int_{\Omega} \psi(\mathbf{x}-\mathbf{y}) d \Omega} \int_{\Omega} \psi(\mathbf{x}-\mathbf{y}) \kappa^{\Omega}(\mathbf{y}) d \Omega
$$

where $\mathbf{y}$ is a typical point of the body $\Omega_{1}$ and the weight function $\psi(\mathbf{x})$ is set as:

$$
\psi(\mathbf{x})=\left\langle 1-\frac{\|\mathbf{x}-\mathbf{y}\|^{2}}{R^{2}}\right\rangle_{+}
$$

with $R$ the radius of the nonlocal integration domain and the symbol $\langle\bullet\rangle_{+}$denoting the positive part of the number $\bullet$. The evolution of the tensile damage parameter is governed by an exponential nonlocal law, set as:

$$
D_{t}^{\Omega}=\max _{\text {history }}\left\{\min \left\{1, \tilde{D}_{t}^{\Omega}\right\}\right\} \quad \tilde{D}_{t}^{\Omega}=\frac{\bar{\varepsilon}_{e q}^{\Omega}-\varepsilon_{0} e^{-k\left(\bar{\varepsilon}_{e q}-\varepsilon_{0}\right)}}{\bar{\varepsilon}_{e q}^{\Omega}}
$$

with $\bar{\varepsilon}_{e q}^{\Omega}(\mathbf{x})$ the equivalent nonlocal strain, evaluated at the point $\mathbf{x}$ as:

$$
\bar{\varepsilon}_{e q}^{\Omega}(\mathbf{x})=\frac{1}{\int_{\Omega} \psi(\mathbf{x}-\mathbf{y}) d \Omega} \int_{\Omega} \psi(\mathbf{x}-\mathbf{y}) \varepsilon_{e q}^{\Omega}(\mathbf{y}) d \Omega
$$

and $\varepsilon_{e q}^{\Omega}$ the equivalent strain introduced as [11]: 


$$
\varepsilon_{e q}^{\Omega}=\sqrt{\left\langle\varepsilon_{1}\right\rangle_{+}^{2}+\left\langle\varepsilon_{2}\right\rangle_{+}^{2}}
$$

with $\varepsilon_{1}$ and $\varepsilon_{2}$ the local principal strains.

Moreover, the following condition is introduced:

$$
D_{t}^{\Omega} \geq D_{c}^{\Omega}
$$

in order to prescribe that the damage in tension should not be lower than the damage in compression.

\section{Interface damage model without coupling}

A phenomenological interface model based on the micromechanical idea, developed in [13] and [14], is proposed. The displacement fields of the two joined bodies are denoted as $\mathbf{u}^{1}$ and $\mathbf{u}^{2}$, while the relative displacement at the typical point $\mathbf{x}^{\mathfrak{I}}$ on the interface $\mathfrak{I}$ is defined as $\mathbf{s}^{\mathfrak{I}}\left(\mathbf{x}^{\mathfrak{I}}\right)=\mathbf{u}^{1}\left(\mathbf{x}^{\mathfrak{I}}\right)-\mathbf{u}^{2}\left(\mathbf{x}^{\mathfrak{I}}\right)$.

At the micromechanical level, the representative area at the point $\mathbf{x}^{\mathfrak{J}}$ is considered; in the representative area microcracks could be present, so that it can be modeled in a simplified form splitting the representative area in two parts: the undamaged and damaged part.

The damage parameter $D^{\mathfrak{\Im}}$ is introduced as the ratio between the damaged area with respect to the reference area; it can vary from zero to one: $D^{\mathfrak{s}}=0$ corresponds to the undamaged state (no microcarcks are present in the representative area), while $D^{\mathfrak{I}}=1$ corresponds to the completely damaged state (the representative area is completely cracked). The stress-relative displacement relationship is formulated:

$$
\boldsymbol{\sigma}^{\mathfrak{I}}=\mathbf{K}^{\mathfrak{I}}\left[\mathbf{s}^{\mathfrak{I}}-D^{\mathfrak{I}}\left(\mathbf{c}^{\mathfrak{I}}+\mathbf{p}^{\mathfrak{I}}\right)\right]
$$

where $\mathbf{K}^{\mathfrak{J}}$ is the interface stiffness matrix, $\mathbf{c}^{\mathfrak{J}}$ is the unilateral contact vector and $\mathbf{p}^{\mathfrak{J}}$ is the sliding friction vector.

A local coordinate system on the interface $\left(x_{T}, x_{N}\right)$, where the indices ${ }_{N}$ and ${ }_{T}$ indicate the normal and the tangential directions of the interface, respectively, is introduced. In this coordinate system, the stiffness matrix, the unilateral contact vector and the sliding friction vector are represented as:

$$
\mathbf{K}^{\mathfrak{J}}=\left[\begin{array}{cc}
K_{N} & 0 \\
0 & K_{T}
\end{array}\right] \quad \mathbf{c}^{\Im}=H\left(s_{N}\right)\left\{\begin{array}{c}
s_{N} \\
0
\end{array}\right\} \quad \mathbf{p}^{\Im}=\left\{\begin{array}{c}
0 \\
p_{T}
\end{array}\right\}
$$

In order to define the evolution of the inelastic slip relative displacement, the stress given in Eq. (13) is rewritten in the following form:

$$
\boldsymbol{\sigma}^{\mathfrak{I}}=\boldsymbol{\sigma}_{d}^{\mathfrak{I}}+\left(1-D^{\mathfrak{I}}\right) \mathbf{K}^{\mathfrak{I}}\left(\mathbf{c}^{\mathfrak{I}}+\mathbf{p}^{\mathfrak{I}}\right)
$$

defining the contact-frictional stress $\boldsymbol{\sigma}_{d}^{\mathfrak{J}}$ as:

$$
\boldsymbol{\sigma}_{d}^{\mathfrak{I}}=\mathbf{K}^{\mathfrak{I}}\left(\mathbf{s}^{\mathfrak{I}}-D^{\mathfrak{J}}\left(\mathbf{c}^{\mathfrak{I}}+\mathbf{p}^{\mathfrak{I}}\right)\right)
$$

It is assumed that the stress $\boldsymbol{\sigma}_{d}^{\mathfrak{J}}$ governs the evolution of the inelastic slip relative displacement. In particular, the classical Coulomb yield function is introduced:

$$
\phi\left(\boldsymbol{\sigma}_{d}^{\mathfrak{I}}\right)=\mu\left\langle\sigma_{d N}\right\rangle_{-}+\left|\sigma_{d T}\right|=\mu \sigma_{d N}+\left|\sigma_{d T}\right|
$$

where $\mu$ is the friction coefficient and the symbol $\left\langle\sigma_{d N}\right\rangle_{-}$denotes the negative part of the contact-frictional stress. The following non-associated flow rule is considered for the evolution of the components of the vector $\mathbf{p}^{\Im}$ : 


$$
\dot{\mathbf{p}}^{\mathfrak{I}}=\dot{\lambda}\left\{\begin{array}{c}
0 \\
\frac{d \phi}{d \sigma_{d T}}
\end{array}\right\}=\dot{\lambda}\left\{\begin{array}{c}
0 \\
\frac{\sigma_{d T}}{\left|\sigma_{d T}\right|}
\end{array}\right\}
$$

together with the Khun-Tucker conditions:

$$
\dot{\lambda} \geq 0, \quad \phi\left(\boldsymbol{\sigma}_{d}^{\mathfrak{I}}\right) \leq 0, \quad \dot{\lambda} \phi\left(\boldsymbol{\sigma}_{d}^{\mathfrak{I}}\right)=0
$$

It can be remarked that the frictional problem can be activated only when the damage is greater than zero. In fact, only in this case the microcracks, in which the unilateral and friction effects can occur, are present at the interface.

About the evolution of the damage parameter $D^{\mathfrak{J}}$, a model which accounts for the coupling of mode I of mode II of fracture is considered. In fact, the two quantities $\eta_{N}$ and $\eta_{T}$, defined as the ratios between the first cracking relative displacement $s_{N}^{0}$ and $s_{T}^{0}$ and the full damage relative displacement $s_{N}^{f}$ and $s_{T}^{f}$, are introduced:

$$
\eta_{N}=\frac{s_{N}^{0}}{s_{N}^{f}}=\frac{s_{N}^{0} \sigma_{N}^{0}}{2 G_{c N}}, \quad \eta_{T}=\frac{s_{T}^{0}}{s_{T}^{f}}=\frac{s_{T}^{0} \sigma_{T}^{0}}{2 G_{c T}}
$$

where $\sigma_{N}^{0}$ and $\sigma_{T}^{0}$ are the peak stresses corresponding to the first cracking relative displacement and $G_{c N}$ and $G_{c T}$ are the specific fracture energies in mode I and mode II, respectively. Then, the parameter $\eta$, which relates the two modes of fracture, is defined as follows:

$$
\eta=\frac{\left\langle s_{N}\right\rangle_{+}^{2}}{\|\tilde{\mathbf{s}}\|^{2}} \eta_{N}+\frac{s_{T}{ }^{2}}{\|\tilde{\mathbf{s}}\|^{2}} \eta_{T}
$$

where $\tilde{\mathbf{s}}=\left\{\left\langle s_{N}\right\rangle_{+} s_{T}\right\}^{T}$. The relative displacement ratios are introduced as:

$$
Y_{N}=\frac{\left\langle s_{N}\right\rangle_{+}}{s_{N}^{0}} \quad Y_{T}=\frac{s_{T}}{s_{T}^{0}}
$$

and the equivalent relative displacement ratio is considered:

$$
Y=\sqrt{Y_{N}^{2}+Y_{T}^{2}}
$$

Finally, the damage parameter is assumed to be a function of the history of the relative displacement as follows:

$$
D^{\mathfrak{J}}=\max _{\text {history }}\left\{0, \min \left\{1, \tilde{D}^{\mathfrak{\Im}}\right\}\right\} \quad \tilde{D}^{\mathfrak{J}}=\frac{Y-1}{Y(1-\eta)}
$$

\section{Interface damage models with coupling}

An interface coupled model, obtained considering different ways of coupling the body and the interface damage, is proposed.

Denoting with $D^{I}(\mathbf{x})$ the coupled interface damage evaluated in a point $\mathbf{x}$ of the interface, the coupling between the body damage and the interface damage is performed, in the firs case, ensuring that the interface damage is not lower than the body damage computed on the bond surface [11]:

$$
D^{I}(\mathbf{x})=\max \left\{D^{\Im}(\mathbf{x}), D_{t}^{\Omega}(\mathbf{x})\right\}
$$

In the second case, a representative area $A$ of the interface and, in particular, of the third layer made of cohesive support material, is assumed to be decomposed in two parts, as represented in Fig. 1. In fact, when the body damage occurs, it induces the presence of a microfracture in the representative area of the surface, characterized by a corresponding area 
$A^{\Omega}=D_{t}^{\Omega} A$. Because of the presence of the microcrack, the stress $\boldsymbol{\sigma}^{W}$ in $A^{\Omega}$ is equal to zero if the microcrack is open and it is different from zero when it is closed. In the remaining part of the representative area, characterized by an area $A^{\Im}=\left(1-D_{t}^{\Omega}\right) A$, it is assumed that the mechanical response is governed by the constitutive model described by Eqs. (13)(25). Thus, the overall constitutive response of the coupled interface is obtained as:

$$
\boldsymbol{\sigma}^{I}=D_{t}^{\Omega} \boldsymbol{\sigma}^{W}+\left(1-D_{t}^{\Omega}\right) \boldsymbol{\sigma}^{\Im}
$$

with $\boldsymbol{\sigma}^{\mathfrak{3}}$ given by Eq. (13) and $\boldsymbol{\sigma}^{W}$ defined as:

$$
\boldsymbol{\sigma}^{W}=\left\{\begin{array}{c}
K_{N}^{\mathfrak{I}}\left(s_{N}^{\mathfrak{I}}-c_{N}^{\mathfrak{I}}\right) \\
0
\end{array}\right\}
$$

where $c_{N}^{\mathfrak{I}}$ the normal component of $\mathbf{c}^{\mathfrak{3}}$ defined by the second equation of the relations (14).

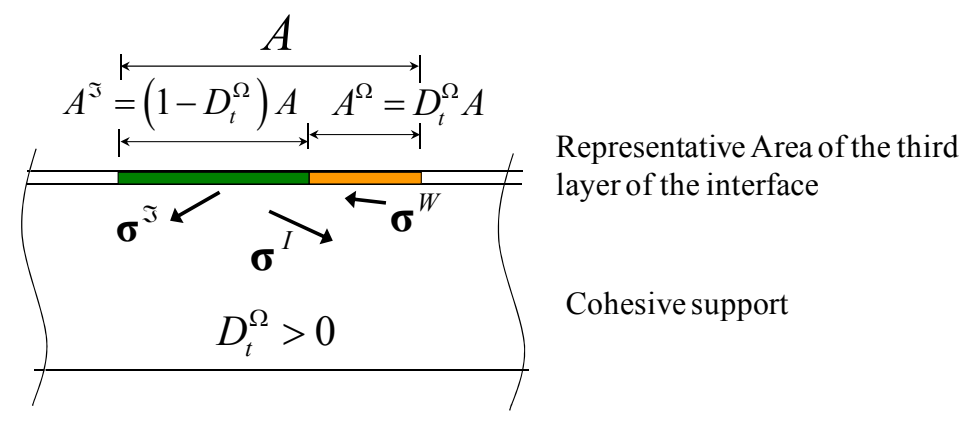

Figure 1: Representative area of the third layer of the interface.

\section{NUMERICAL APPLICATIONS}

$\mathrm{N}$

umerical procedures for solving the equations governing the mechanical response of the body-interface nonlocal damage models, described in the previous section, are developed. body andinterface models. In particular, the time integration is performed adopting a backward-Euler implicit procedure. The proposed numerical procedure is implemented in the finite element code FEAP [15]. In particular, two dimensional plane stress four node quadrilateral elements are adopted to model the bodies $\Omega_{1}$ and $\Omega_{2}$ and four node interface elements are developed to model the interface $\mathfrak{I}$.

Some numerical applications are carried out in order to assess the efficiency of the proposed coupled nonlocal damage interface-body model in describing the detachment phenomenon of the FRP reinforcement from the cohesive material.

In particular, in the following applications Model 1 indicates the interface model, in which the coupling is taken into account assuring that the interface damage is not lower than the body damage computed on the bond surface, while Model 2 indicates the formulation developed on the basis of a simplified micromechanical analysis.

The properties of the materials adopted in the numerical applications are set on the basis of the experimental detachment tests performed on masonry elements reinforced with FRP [16]:

- $\quad$ Body $\Omega_{1}$

$$
\begin{array}{rlrl}
E_{1} & =15300 \mathrm{MPa} & v_{1}=0.2 \quad R=15.0 \mathrm{~mm} \\
\varepsilon_{0} & =0.00029 & G_{c} & =0.0095 \mathrm{~N} / \mathrm{mm}^{2} \\
\varepsilon_{u} & =-0.003 & \sigma_{y} & =-20 \mathrm{MPa}
\end{array}
$$

- $\quad$ Body $\Omega_{2}$

$$
E_{2}=160000 \mathrm{MPa} \quad v_{2}=0.3
$$


- Interface $\mathfrak{J}$

$$
\begin{array}{ll}
K_{N}=K_{T}=270 \mathrm{~N} / \mathrm{mm}^{3} & \mu=0.5 \\
\sigma_{N}^{0}=\sigma_{T}^{0}=4.7 \mathrm{MPa} & G_{c N}=G_{c T}=0.34 \mathrm{~N} / \mathrm{mm}
\end{array}
$$

where $E_{1}, v_{1}, E_{2}$ and $v_{2}$ are the Young modulus and the Poisson coefficient of the body $\Omega_{1}$ and $\Omega_{2}$, respectively. In particular, first a simple tensile test is performed to show how the response of the interface can be significantly influenced by the damage occurring in the body $\Omega_{1}$. Then, the maximum detachment force is evaluated for different values of the adhesion lengths and of the initial values of the body damage.

\section{Tensile test}

The geometry and loading condition of the scheme considered to perform the tensile test are shown in Fig. 2. The geometrical parameters are $b=500 \mathrm{~mm} \quad h=49 \mathrm{~mm}$ and an unit thickness is adopted.

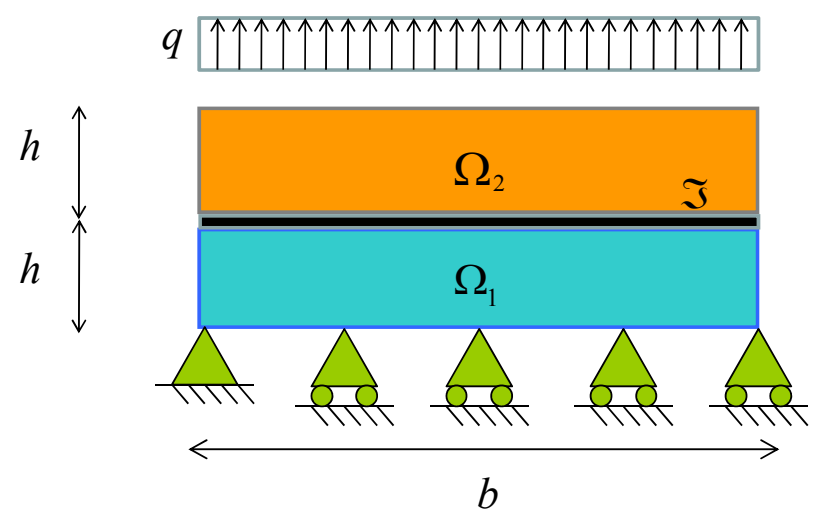

Figure 2: Scheme of the uniaxial test.

In order to investigate the influence of the damaging behavior of the body $\Omega_{1}$ on the tensile mechanical response of the interface and, as a consequence, of the whole structure, three analyses are developed considering different values of the initial threshold damage strain $\varepsilon_{0}$ and keeping constant the fracture energy $G_{c}$; in particular it is set:

Case 1: $\varepsilon_{0}=0.00016$

Case 2: $\varepsilon_{0}=0.00026$

Case 3: $\varepsilon_{0}=0.00036$

The three analyses are performed considering as interface model the two coupled damage approaches previously presented (Model 1 and Model 2).

In Fig. 3 and Fig. 4, the numerical response obtained adopting the Model 1 and the Model 2 are shown. The results reported in the graphics of these figures are plotted with a dotted line for Case 1, with a dashed line for the Case 2 and with a solid line for the Case 3. Furthermore, the average tensile stress is introduced as $\bar{\sigma}=q$ and the average strain in the body $\Omega_{1}$ is set as $\bar{\varepsilon}=\Delta v / h$ with $\Delta v$ the relative vertical displacement between the two opposite edges of the body $\Omega_{1}$. The computations are performed adopting the arc-length technique and considering the relative normal displacement $s_{N}$ at the interface as control parameter.

With reference to Fig. 3, it can be noted that in the Case 3 the mechanical response of the structure is strongly influenced only by the softening behavior of the interface as in this analysis the damage does not occur in the body. In fact, the tensile interface response is equal to the constitutive interface law and the body is subject to the elastic unloading when the interface starts to damage. In the other two cases, the tensile mechanical response of the structure depends on the coupling of the body and interface damage. In fact, after the achievement of the peak stress, which coincides with the tensile strength of the body, the softening branch depends on the evolution of the damage in the body until the interface damage, governed by the relative displacement, becomes higher than the body one at the interface. At this point of the analysis the softening tensile response is due to the development of the interface damage governed by the relative displacement. 

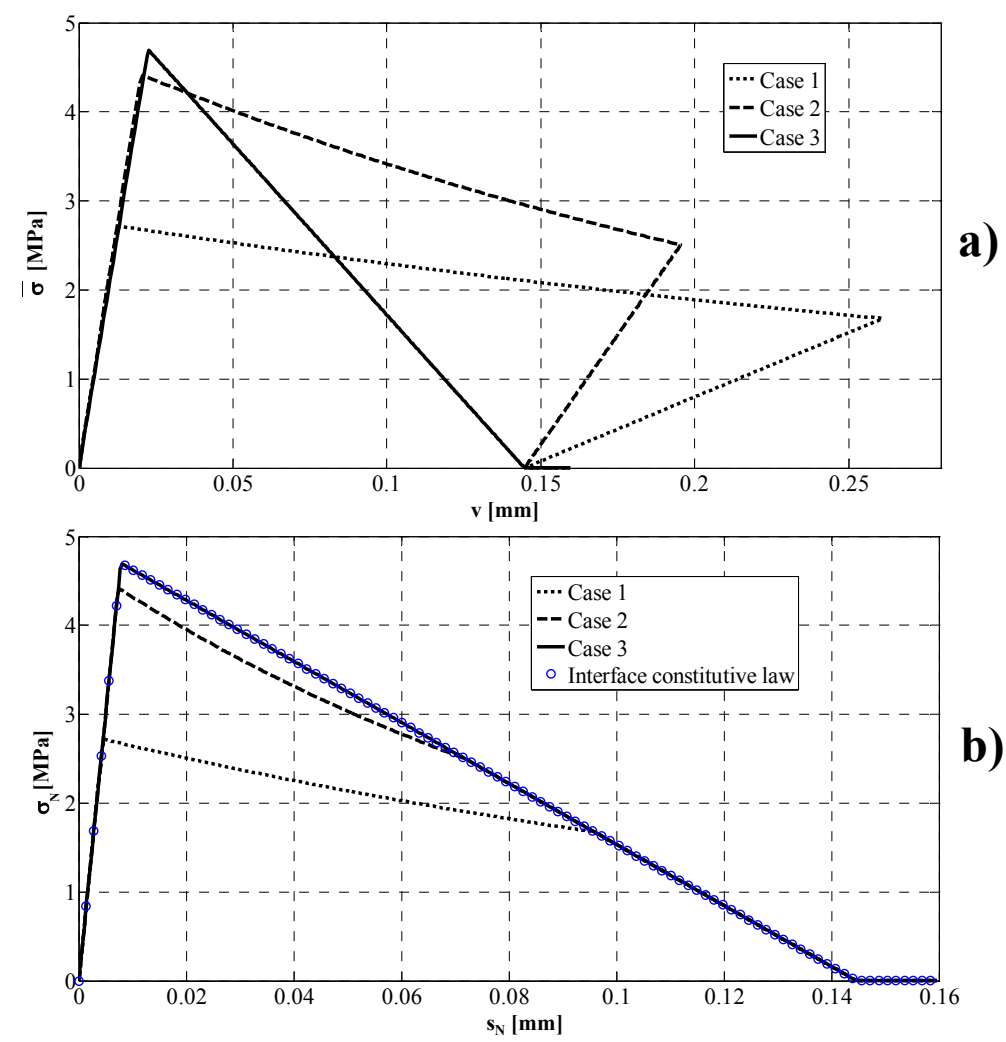

b)

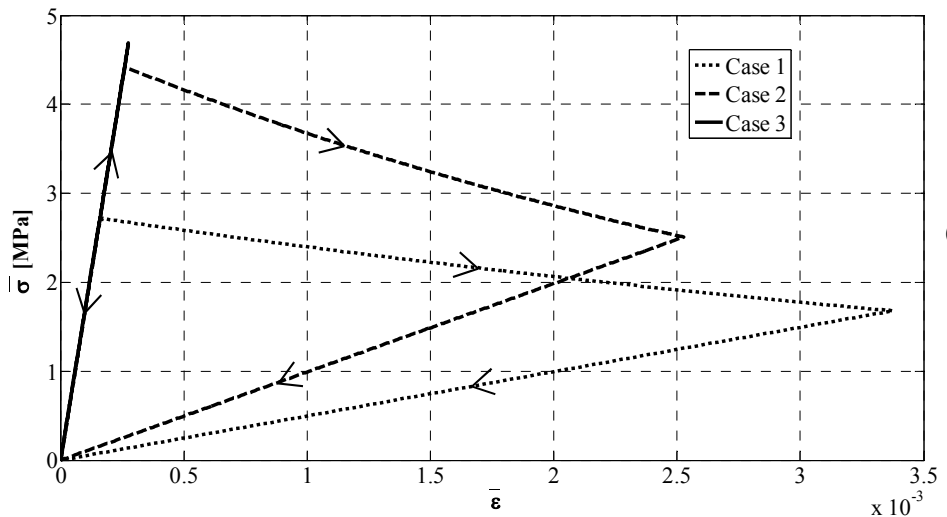

c)

Figure 3: Numerical results obtained adopting the Model 1. a) Mechanical response of whole structure; b) Mechanical behavior of interface; c) Body tensile response.

From Fig. 4, it is observed that in the Case 3 the body does not develop damage and for this reason the softening response of the mechanical system depends only on the evolution of the damage interface. With reference to Fig. 3 and Fig. 4, it appears evident that in the Case 3 the analyses, performed adopting the proposed coupled interface formulations (Model 1 and Model 2), lead to the same numerical results. In the Case 1 and in the Case 2, the body damage occurs before the interface one; the maximum tensile stress is lower than the value obtained in the Case 3 and it is equal to the tensile strength of the body, as it is achieved in the Model 1. In the Case 1 and 2 the softening response, obtained adopting the Model 1 and 2, presents some significant differences. In fact, the results carried out adopting the Model 1, show that the softening behavior is strongly influenced by the evolution of the body damage until the interface damage becomes higher than the body one. From this point of the analysis, the body damage does not increase anymore and the softening behavior is only governed by the evolution of the interface damage. On the other hand, in the results obtained considering the Model 2, the softening behavior is strongly influenced by the body damage during the whole detachment process, also when the interface damage becomes to develop and the body damage does not evolve anymore. Thus, the degradation process results faster for the Model 2 than for the Model 1. 


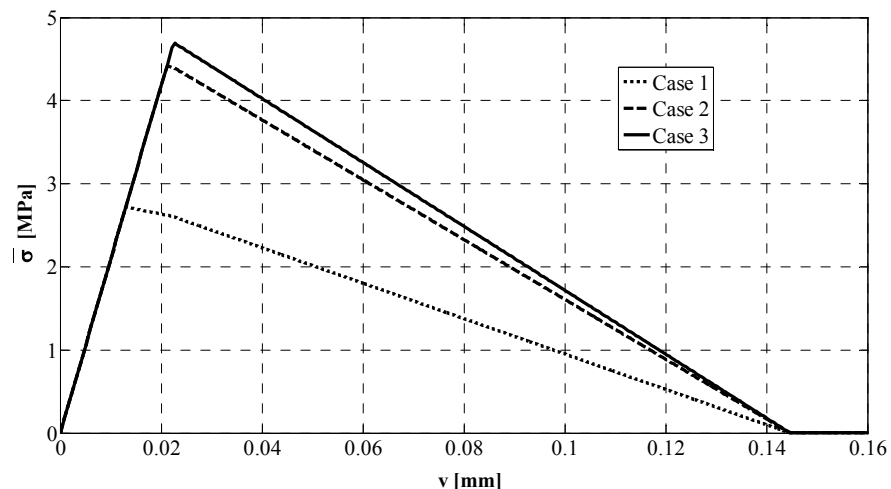

a)

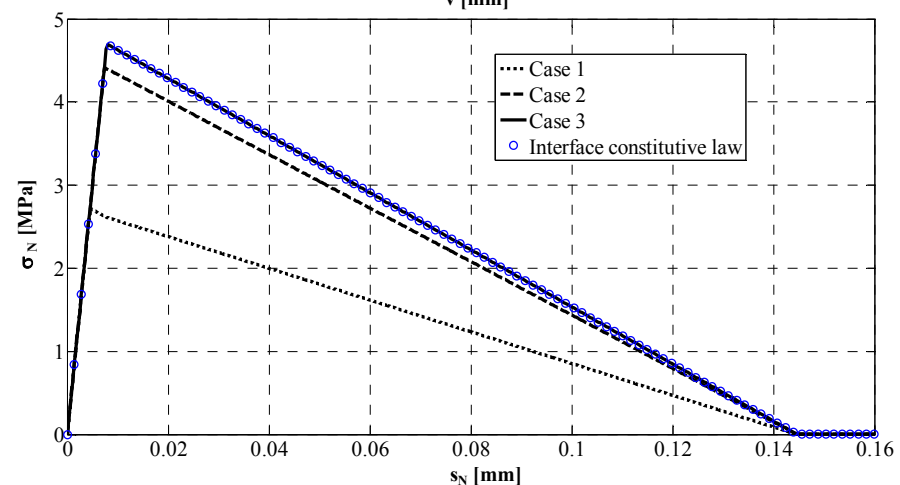

b)

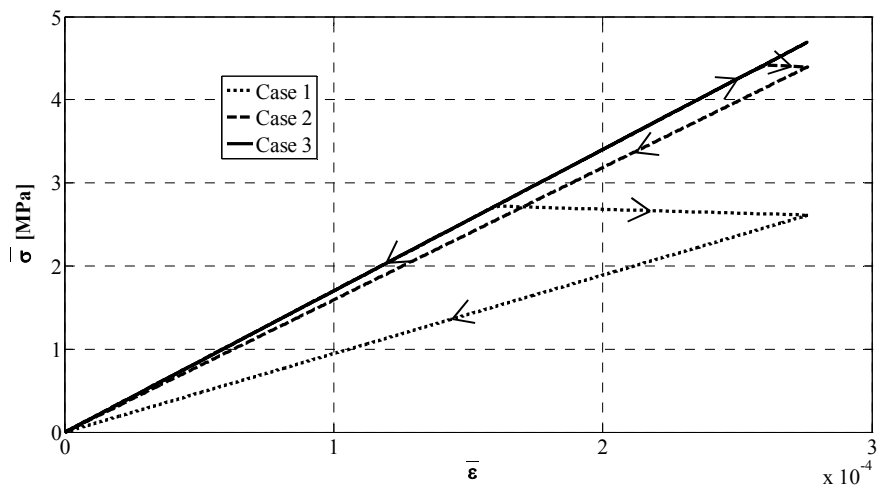

c)

Figure 4: Numerical results obtained adopting the Model 2. a) Mechanical response of whole structure; b) Mechanical behavior of interface; c) Body tensile response.

\section{Maximum decohesion force}

The computations are performed considering the scheme and the geometry illustrated in Fig. 5. The FRP laminate (body $\Omega_{2}$ ) is bonded to a masonry support (body $\Omega_{1}$ ) made of two clay bricks separated by an unitary layer of mortar. The FRP laminate is subjected to tensile loading.

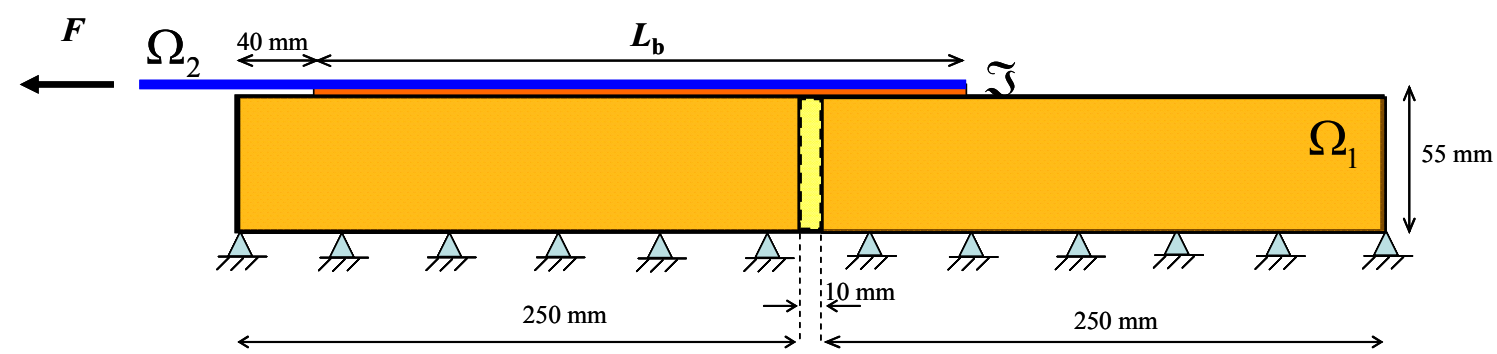

Figure 5: Scheme of the FRP-masonry brick detachment test. 
All computations are developed assuming two interface damage models: the uncoupled model, which does not take into account the interaction between body and interface degradation, and the coupled one, in which the body damage influences the interface damage according to the formulation developed in the Model 1.

In Fig. 6 the value of $F_{\max }$ is plotted versus the adhesion length $L_{b}$. Note that each curve is denoted by a symbol made of a letter and a number. The letter $U$ is used to indicate that the analysis is performed adopting the uncoupled damage model, while the letter $\mathrm{C}$ is used to characterize the analysis developed with the coupled damage theory (Model 1). The number near the letter indicates the initial damage level uniformly assigned at the body $\Omega_{1}$. In particular, the number 1,2 , 3 , and 4 corresponds to the damage value equal to $0,0.5,0.7$, and 0.9 , respectively.

The numerical results reported in Fig. 6 emphasize that, increasing the adhesion length $L_{b}$, the value of $F_{\max }$ grows till the optimal adhesion length $L_{e}$ is reached, after which $F_{\max }$ remains constant.

In particular, from the type $\mathrm{U}$ curves marked by the discontinuous line, it can be noted that:

$\checkmark$ for higher values of the damage state of the body $\Omega_{1}$ the optimal adhesion length $L_{e}$ increases;

$\checkmark$ for higher values of the damage state of the body $\Omega_{1}$ the maximum value of $F_{\max }$ is quite constant and, in some cases, it tends to increase;

$\checkmark$ for very high values of the damage state of the body $\Omega_{1}$ the maximum value of $F_{\max }$ decreases.

While the first result is absolutely expected, the second one appears physically unacceptable, as it implies that even if the support material is more damaged, equal or higher values of the forces can be transmitted from $\Omega_{2}$ to $\Omega_{1}$. On the contrary, only when the damage level of the body $\Omega_{1}$ becomes very high the force decreases. This strange effect is due to the uncoupled damage evolution of the body and of the interface damage state.

With reference to the all type $\mathrm{C}$ curves marked by the solid line, the following observations can be remarked:

$\checkmark$ for higher values of the damage state of the body $\Omega_{1}$ the optimal adhesion length $L_{e}$ increases, as in the case of the uncoupled model;

$\checkmark$ for higher values of the damage state of the body $\Omega_{1}$ the maximum value of $F_{\max }$ decreases.

This last result appears much more reasonable and, as a consequence, more reliable with respect to the one obtained adopting the uncoupled damage model, as it does not suffer from the physical unacceptable effect found in the uncoupled one.

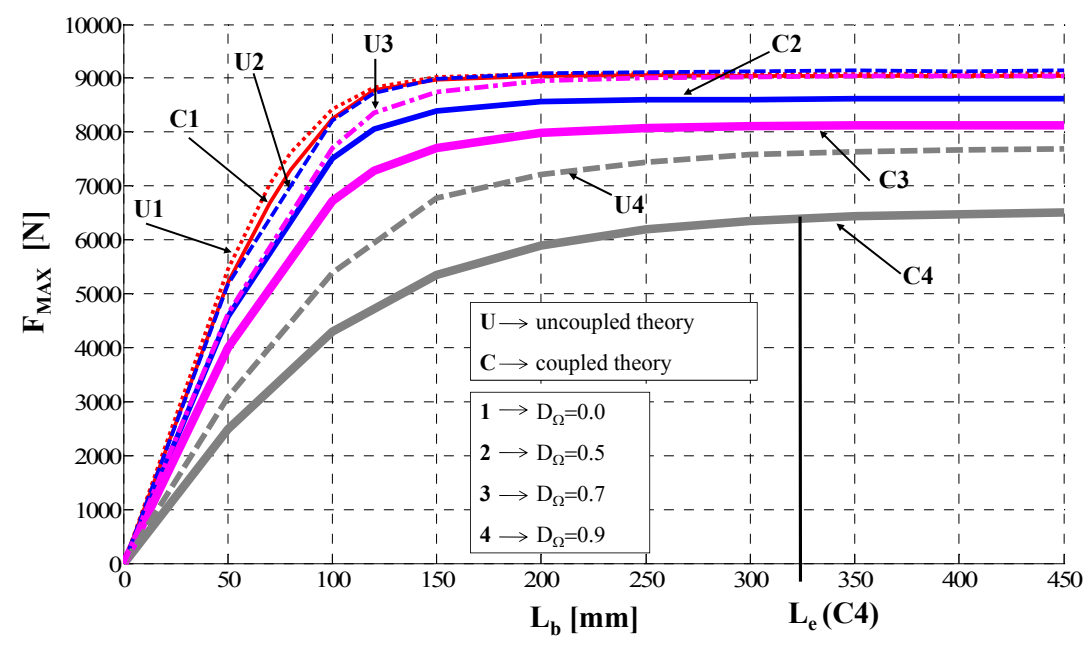

Figure 6: Decohesion force $F_{\max }$ versus adhesion length $L_{b}$.

\section{CONCLUSIONS}

$\mathrm{I}$ $\mathrm{n}$ conclusion, it can be remarked that the two different ways of coupling the body and the interface damage present significant differences in the numerical applications. In fact, the results carried out adopting the Model 1, show that the softening behavior is strongly influenced by the evolution of the body damage until the interface damage becomes higher than the body one. From this point of the analysis, the body damage does not increase anymore and the 
softening behavior is only governed by the evolution of the interface damage. On the other hand, in the results obtained considering the Model 2, the softening behavior is strongly influenced by the body damage during the whole detachment process, also when the interface damage becomes to develop and the body damage does not evolve anymore. Thus, the degradation process results faster for the Model 2 than for the Model 1.

Moreover, the numerical application show also the differences between the response of the coupled and uncoupled interface model. In particular, the results obtained using the coupled model appears much more reasonable and, as a consequence, more reliable with respect to the one obtained adopting the uncoupled damage model, as it does not suffer from the physical unacceptable effect found in the uncoupled one.

\section{REFERENCES}

[1] N. Plevris, T.C. Triantaffilou, D. Veneziano, Journal of Structural Engineering ASCE 121, (1995) 1037.

[2] T.C. Triantafillou, M.N. Fardis, Materials and Structures, 30 (1997) 486.

[3] M. R. Valluzzi, M. Valdemarca, C. Modena, Journal of composites for constructions ASCE (2001) 163.

[4] S. Marfia, E. Sacco, International Journal of Solids and Structures, 38 (2001) 4177.

[5] N. Galati, G. Tumialan, A. Nanni, Construction and Building Materials, 20 (2006) 101.

[6] B. Ferracuti, M. Savoia, C. Mazzotti, Composites: Part B, 37 (2006) 356.

[7] F. Freddi, M. Savoia, Engineering Fracture Mechanics, 75 (2008) 1666.

[8] E. Grande, G. Milani, E. Sacco, Engineering Structures, 30 (2008) 1842.

[9] F. Fouchal, F. Lebon, I. Titeux, Construction and Building Materials, 23 (2009) 2428.

[10] F. Freddi, M. Fremond, Journal of Mechanics of Materials and Structures, 7 (2006) 1205.

[11] S. Marfia, E. Sacco, J. Toti, A coupled interface-body nonlocal damage model for FRP strengthening detachment, In print on Computational Mechanics (2011).

[12] Mazars J, Pijaudier-Cabot G Continuum damage theory: application to concrete. Journal of Engineering Mechanics, ASCE, 115 (1989) 345.

[13] G. Alfano, E. Sacco, International Journal For Numerical Methods in Engineering, 68 (2006) 542.

[14] E. Sacco, J. Toti, International Journal for Computational Methods in Engineering Science and Mechanics, 11 (2010) 354.

[15] O.C. Zienkiewicz, R. L. Taylor The Finite Element Method, 4th edn. McGraw-Hill, London, (1991).

[16] E. Grande, M. Imbimbo, E. Sacco, Journal of Composites Part B: Engineering, 42 (2011) 330. 